\title{
Nanomechanics of a hydrogen molecule suspended between two equally charged tips
}

\author{
W. Schattke $\odot,{ }^{1,2, *}$ T. Frederiksen $\odot,{ }^{1,3}$ M. A. Van Hove $\odot,{ }^{4}$ and R. Díez Muiño $\odot^{1,5}$ \\ ${ }^{1}$ Donostia International Physics Center (DIPC), P. Manuel de Lardizabal 4, 20018 San Sebastián, Spain \\ ${ }^{2}$ Institut für Theoretische Physik und Astrophysik der Christian-Albrechts-Universität, Leibnizstraße 15, 24118 Kiel, Germany \\ ${ }^{3}$ IKERBASQUE, Basque Foundation for Science, E-48013 Bilbao, Spain \\ ${ }^{4}$ Department of Physics and Institute of Computational and Theoretical Studies (ICTS), Hong Kong Baptist University, \\ 224 Waterloo Road, Kowloon, Hong Kong \\ ${ }^{5}$ Centro de Física de Materiales CFM (CSIC-UPV/EHU)-Materials Physics Center MPC, \\ P. Manuel de Lardizabal 5, 20018 San Sebastián, Spain
}

(Received 24 December 2019; revised manuscript received 4 March 2020; accepted 21 April 2020; published 26 May 2020)

\begin{abstract}
The geometric configuration and energy of a hydrogen molecule centered between two point-shaped tips of equal charge are calculated with the variational quantum Monte Carlo (QMC) method without the restriction of the Born-Oppenheimer (BO) approximation. The ground-state nuclear distribution, stability, and low-vibrational excitations are found to deviate significantly from the BO treatment based on a potential energy surface obtained with the same QMC accuracy. The quantum-mechanical distribution of the molecular axis direction and the bond length at a subnanometer level is fundamental for understanding nanomechanical dynamics with embedded hydrogen. The cylindrical symmetry of the tip arrangement yields a uniform azimuthal distribution of the molecular axis vector relative to the tip-tip axis. For fixed tip separation, the QMC sampling shows that the polar angle distribution of the molecular axis is centered around the equatorial plane for positive tip charge (transverse alignment) and around the tip-tip direction for negative tip charge (bridge alignment). These deviations from spherical symmetry are magnified as the tip-tip distance decreases. Our results thus show that the molecular orientation in the junction can be controlled by the tip charge and separation, suggesting an application in the field of molecular machines.
\end{abstract}

DOI: 10.1103/PhysRevB.101.195436

\section{INTRODUCTION}

Growing interest in nanomechanical properties is reflected in a wide range of research, from the chemistry of synthesizing suitable nanoscale molecules to the physics of manipulating those objects, whether isolated or adsorbed on surfaces and nanostructures. "Supramolecular chemistry" [1] dates back to the work on molecular switches through the synthesis of catenanes and rotaxanes [2] leading to nanoscale machines such as artificial molecular pumps [3], molecular wheels, or even "nanocars" [4-6].

In addition to reaction chemistry methods, one can use an external physical tool for manipulation, e.g., by interaction with the chemical object via electromagnetic fields. There is a vast literature on these topics. Here we cite only a few examples that come closer to our investigations: Nanocavities built by atomic assembly at a metal surface with a scanning tunneling microscope (STM) [7-9] as well as nanogaps formed with the mechanically controllable break junction technique [10-13] are both able to hold in place, bind, and subsequently excite a hydrogen molecule $\left(\mathrm{H}_{2}\right)$ to perform the desired motion, e.g., vibration or rotation. At low temperatures, the interaction between the externally applied electronic current and a trapped molecule can be revealed as inelastic variations

*Corresponding author: schattke@theo-physik.uni-kiel.de in the second derivative of the current-voltage characteristics when the bias voltage coincides with an excitation energy of the junction. As a captive $\mathrm{H}_{2}$ molecule can be accurately accessed by both experiment and $a b$ initio theory, it can serve as the basis for complex nanomechanics.

In light of these developments, we investigate a system that combines simplicity at an $a b$ initio level with feasibility at the nanoscale: $\mathrm{a}_{2}$ molecule bridging two STM-like tips, each with an equal point charge. This design enables external control of the molecule by changing charge and distance. Fundamental work on this molecule ranges from high-precision calculations $[14,15]$ to high-precision measurement, such as, e.g., highly accurate vibrational determinations [16]. It extends as well to the challenge of examining the generally used Born-Oppenheimer assumption [17-19]. For instance, the first vibration energy of $\mathrm{H}_{2}$ is lowered through nonadiabatic corrections from the $\mathrm{BO}$ value of $0.51620 \mathrm{eV}$ by about $0.03 \%$ to almost exactly yield the experimental result of $0.51592 \mathrm{eV}$. Among all atoms and molecules, the light $\mathrm{H}_{2}$ is most sensitive to nonadiabatic effects that require us to coherently consider the quantum-mechanical nuclear motion within an electronic calculation. These effects will also express themselves in the model considered here. Furthermore, they could be enhanced in a clamped system by both nuclear motion confined by external forces and a fixed center of mass. Specifically, one would expect an increase of the zero-point energy, a weakening of the $\mathrm{H}_{2}$-bond, and a tightening of the binding by the tips in the 
adiabatic case. By contrast, the nonadiabatic system would release some of that binding energy by the free floating of the nuclei with the electron motion, thereby yielding a rather unpredictable result of the total effect. However, we believe that the small size of the nonadiabatic correction in the case of the free molecule cannot be transferred to the clamped situation.

The ground-state energy and the first vibrational excitation are chosen here as the main quantities to probe the response of the hydrogen nuclear motion upon the action of an external static electric field. This system has more general importance beyond the aforementioned examples and their detailed realizations. The molecule can be detached, for instance, from the STM tips and placed in another stable electrostatic field environment produced by a molecular neighborhood of similar symmetry, which may allow us to study the processes that underlie reaction chemistry.

The rotational degrees of freedom of the free molecule in unperturbed space will be restricted by an external perturbation and so will the zero-point energy, thus affecting its interaction with the surroundings. For instance, the probability distribution of the molecular orientation in space is one of the keys for the reactivity with neighboring molecules. The knowledge of the molecule's behavior under the influence of charged tips could also help to orient controllably the molecular axis into some preferred direction. Although the discussion here focuses on a two-tip/one-molecule system, it might be used in a first step toward more complicated and extended nanomechanical systems as an example for an incorporated hydrogen molecule. Our example serves to understand the physical dynamics of the $\mathrm{H}_{2}$ molecule in an external electrostatic field that does not break its bond.

Because of the demanding computational costs of the QMC method, our idealized configuration still lacks some material specificity at this stage. Nevertheless, it is related to realizable subnanoscale mechanical physical arrangements, while the precision gained here is needed to detect or rule out tiny effects that may arise beyond the $\mathrm{BO}$ approximation or beyond more macroscopic modeling.

\section{PHYSICAL SYSTEM AND COMPUTATIONAL METHOD}

\section{A. Hydrogen molecule between two tips}

Our system consists of two tips represented as point charges $Q$ of equal sign, i.e., ++ or -- , positioned at a distance $D$ apart. The externally controllable parameters $Q, D$ are varied within the range of a few tenths of a.u. for $Q$ and from 4 a.u. to very large distances for $D$. The setup is shown schematically in Fig. 1. All other parameters appearing in the calculations are $a b$ initio optimized. The point charges could have been generalized by replacing them with fictitious $\mathrm{H}$ atoms without gaining much more insight with respect to the goal of understanding the reaction of the molecule to changing the tip-tip distance and the charge. For simplicity, we consider the center of nuclear masses to be at a high symmetry position, i.e., halfway between the tips. This and the exclusion of the opposite (+-) charges is suggestive, as the electrostatic field of the tips acting on the nuclei already has a saddle point

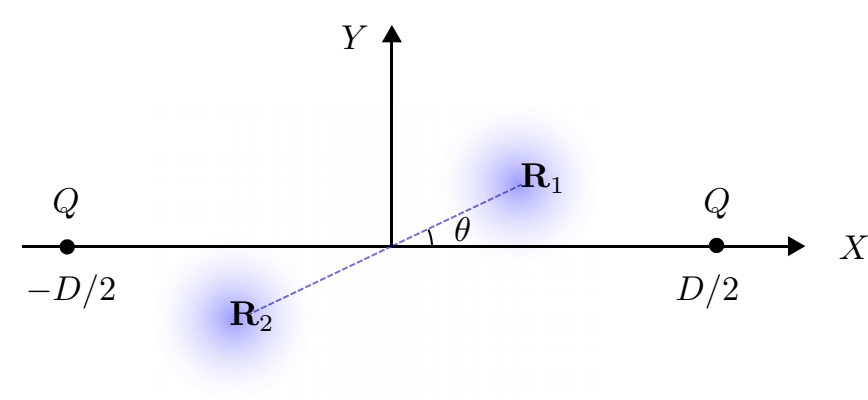

FIG. 1. Setup and coordinate system for a $\mathrm{H}_{2}$ molecule between point charges $Q$ of equal sign, positioned at a distance $D$ apart. The molecular axis, defined by nuclear coordinates $\mathbf{R}_{1}$ and $\mathbf{R}_{2}$, forms a polar angle $\theta$ with the $X$ axis.

potential shape. Thus, the polarized molecule can be expected to attain at least a metastable center-of-mass position.

With one rotation axis and one mirror plane, this centered system is compatible with two pointlike tips of equal charge. It has the highest symmetry, which usually is also the fingerprint of a ground state. We checked the stability or instability at that center by means of the energy of the molecule when it is infinitely distant from the tips. Because of the fixed center-ofmass, the four moving point charges (two electrons and two nuclei) can be quantum mechanically treated with only nine degrees of freedom.

This implies that we must dispense here with charge neutrality, which would characterize normal environments of a $\mathrm{H}_{2}$ molecule, such as in a crystal or a liquid. Oppositely charged tips are also of interest because of the voltage drop and the electric current characteristic in the $\mathrm{H}_{2}$ bridge experiments [7-13]. This will be pursued in later work.

Nevertheless, the situation considered here with equally charged tips presents an interesting variety of physical properties, e.g., the question of whether and how the molecule will dissociate with approaching tips, or whether the molecule will detach as a whole from the tips into the surrounding vacuum. The field that is produced by the tips in this case also stands for other situations with similar local forces at a hydrogen molecule to which one may analogously apply our results regarding stability or vibration frequency.

\section{B. Hamiltonian and wave function}

The total energy $E$ and Hamiltonian $\hat{H}$ comprise the kinetic energy $\hat{T}$, potential energy $\hat{V}$, and interaction energy $\hat{W}$, observables of the two nuclei and the two electrons with six electron and six nucleon degrees of freedom, viz.,

$$
\begin{aligned}
\hat{H} & =\hat{T}_{\mathrm{nu}}+\hat{V}_{\mathrm{nu}}+\hat{T}_{\mathrm{el}}+\hat{V}_{\mathrm{el}}+\hat{W}_{\mathrm{nu}-\mathrm{el}}, \\
& =\hat{T}_{\mathrm{nu}}+\hat{V}^{\mathrm{ad}} \\
\hat{V}^{\mathrm{ad}} & :=\hat{V}_{\mathrm{nu}}+\hat{T}_{\mathrm{el}}+\hat{V}_{\mathrm{el}}+\hat{W}_{\mathrm{nu}-\mathrm{el}}=V^{\mathrm{ad}}\left(\hat{\mathbf{r}}_{i}, \hat{\mathbf{R}}_{j}\right) \\
E & =\langle\Upsilon|\hat{H}| \Upsilon\rangle=\int \Upsilon^{*}\left(\mathbf{r}_{i}, \mathbf{R}_{j}\right) H \Upsilon\left(\mathbf{r}_{i}, \mathbf{R}_{j}\right) d^{6} r d^{6} R .
\end{aligned}
$$

The general wave function $\Upsilon$ can be decomposed as a product of a part $\Phi$, which depends solely on the nucleons' 
positions $\mathbf{R}_{j}, j=1,2$, and a remaining electron part $\Psi$, which contains the positions $\mathbf{r}_{i}, i=1,2$, of the electrons, and also depends on the nucleon positions. For example, the centers of the electronic orbitals represent singularities, and their varying position has a non-negligible influence on the nuclear kinetic energy, in contrast to the simple product ansatz of the BO approximation. Both parts depend on variational parameters $\alpha_{k}$ for the electrons and $\zeta_{l}$ for the nuclei, where the former in turn also depend on the nuclei positions, if the adiabatic approximation is used. The nuclear position dependence is represented by the variable distance vector $\mathbf{R}=\mathbf{R}_{2}-\mathbf{R}_{1}$ between both nuclei, i.e., writing $\alpha_{k}=\alpha_{k}\left(\mathbf{R}_{j}\right)$ with implying the presumed fixed center of mass at zero,

$$
\Upsilon\left(\mathbf{r}_{i}, \mathbf{R}_{j}\right)=\Psi_{\mathrm{el}}\left(\mathbf{r}_{i}, \mathbf{R}_{j} ; \alpha_{k}\right) \Phi_{\mathrm{nu}}\left(\mathbf{R}_{j} ; \zeta_{l}\right), \quad i, j=1,2 .
$$

The notion of the $\mathbf{R}$-dependence in the $\alpha$ parameters refers here to their dependence as a result of an adiabatic optimization where the nuclear positions are fixed during a search for the minimum of the electron energy expectation, disregarding the nuclear wave-function part at this stage. The parameters $\alpha$ then depend on $\mathbf{R}$ and the electron energy yields the potential energy surface of the Born-Oppenheimer (BO) approximation. The nuclear dynamics are introduced after this averaging of the electronic degrees of freedom with a nuclear wave function of the above type.

In contrast, our nonadiabatic treatment fixes these variational parameters in a simultaneous optimization of the electronic and nucleonic degrees of freedom such that the explicit $\mathbf{R}$ notation in the $\alpha$ becomes obsolete. Equation (5) covers the most general eigenfunction of the full electron-nucleon Hamiltonian under a center-of-mass restriction. Below, we sometimes keep the six-dimensional nuclei space for general notation. We emphasize that the nuclear positions are quantum-mechanical variables such that we do not assign fixed values to them.

The highest symmetry compatible with the geometrical arrangement of two tips and a $\mathrm{H}_{2}$ molecule centered between them is described by cylindrical coordinates. The asymptotic case of the free molecule is reached at infinite tip distance and yields spherical symmetry. The $X$-coordinate is taken as the cylinder axis and is associated with the longitudinal motion of the nuclei along the tips' connection line. The ground-state nuclear wave function is written in relative coordinates $\mathbf{R}:=$ $\mathbf{R}_{2}-\mathbf{R}_{1}=(X, Y, Z)$ as a Gaussian distribution

$$
\begin{aligned}
\Phi_{\mathrm{nu}}^{(0)}\left(\mathbf{R}_{1}, \mathbf{R}_{2}\right)= & \exp \left\{-\zeta_{0}\left[\sqrt{\left(X^{2}+Y^{2}+Z^{2}\right)}-R_{0}\right]^{2}\right. \\
& -\zeta_{0 x}\left(|X|-R_{0 x}\right)^{2} \\
& \left.-\zeta_{0 y z}\left[\sqrt{\left(Y^{2}+Z^{2}\right)}-R_{0 y z}\right]^{2}\right\} .
\end{aligned}
$$

The three parameters $R_{0}, R_{0 x}, R_{0 y z}$ are used as centers of the Gaussian ansatz for the spherical, axial, and planar radial dimensions, respectively, instead of a single hydrogen bond length. They are associated with three corresponding parameters for their statistical widths. They represent a variational freedom of in total six nuclear parameters that have to be optimized, in addition to the electronic parameters for the total energy minimum. One may, for instance, propose an ansatz that uses the set of Hermite functions instead of Eq. (6) according to the harmonic oscillator with a ladder of equidis- tant eigenvalues and one single energy-independent Gaussian exponent, but this is too simple. For describing atom-atom interactions, one needs to approximate the asymmetry between close and far distances, e.g., as is accomplished by the Lennard-Jones potential. A harmonic potential fails to do that, even for the low-lying vibrational eigenvalues. The variational functions obtained here can reflect the actual symmetry on the basis of the nuclear positions that are dynamically influenced by the electron positions.

\section{Monte Carlo run and optimization sweep}

The QMC code that we have developed and used (see, e.g., [21]) is organized in the variational mode. We denote by a QMC run the outermost loop in the METROPOLIS algorithm, which consists of MCMAX (typically $10^{9}$ ) steps over randomly sampled positions of both electrons. Such a run is simultaneously performed usually on 24 parallel CPU's with differing initial seeds of the random walk to obtain better statistics. After every 100 moves of the electrons, both protons step randomly with a maximum step size of $1 / 5$ of that of the electronic step size. In extreme cases, it is adapted to the length scale of the wave function. Within a run the observables are calculated together with their statistical data.

An optimization sweep sequentially calculates the observables for all optimization parameters one after the other and on a set of values sufficient to determine a reliable minimum of energy expectation. In addition to the above-mentioned nuclear parameters, we used around 30 electronic parameters that appear in the product of double-zeta Slater-type orbitals with a Jastrow factor of Gaussian-type orbitals. Both of them are formulated within a geminal determinant ansatz [20] to span the variational space. These parameters more or less sensitively influence the energy expectation that is used to accelerate the minimum search. For a more detailed description of the specific parameters of the wave function, the reader is referred to the Supplemental Material [25]. Several sweeps were necessary to obtain a clear signature of a minimum in energy with reasonable variance and with reliable MC statistics. During optimization, intermediate runs are used with length reduced by a factor of 10 with a corresponding loss in statistical accuracy by roughly a factor of 3 for the energy expectation.

The quality of the resulting optimum depends solely on the adequacy of the parametrized Hilbert space. The difference from the BO approach consists in the fact that our wavefunction choice does not factorize into one part depending quantum mechanically only on the electron coordinates, treating the nuclear coordinates as classical variables (or parameters), and another part depending only on the nuclear coordinates. There are many places where the nuclear positions appear in the electronic wave function and have to be treated as quantum-mechanical variables. The orbitals of the determinant as well as those of the Jastrow factor depend on them in an essential way, even producing singularities. We did not consider any implicit functional dependence of the electronic parameters on the nuclear coordinates, though this could principally widen the variational freedom. However, it might be noted that the parameter optimum relies on optimizing them simultaneously and not separately. 


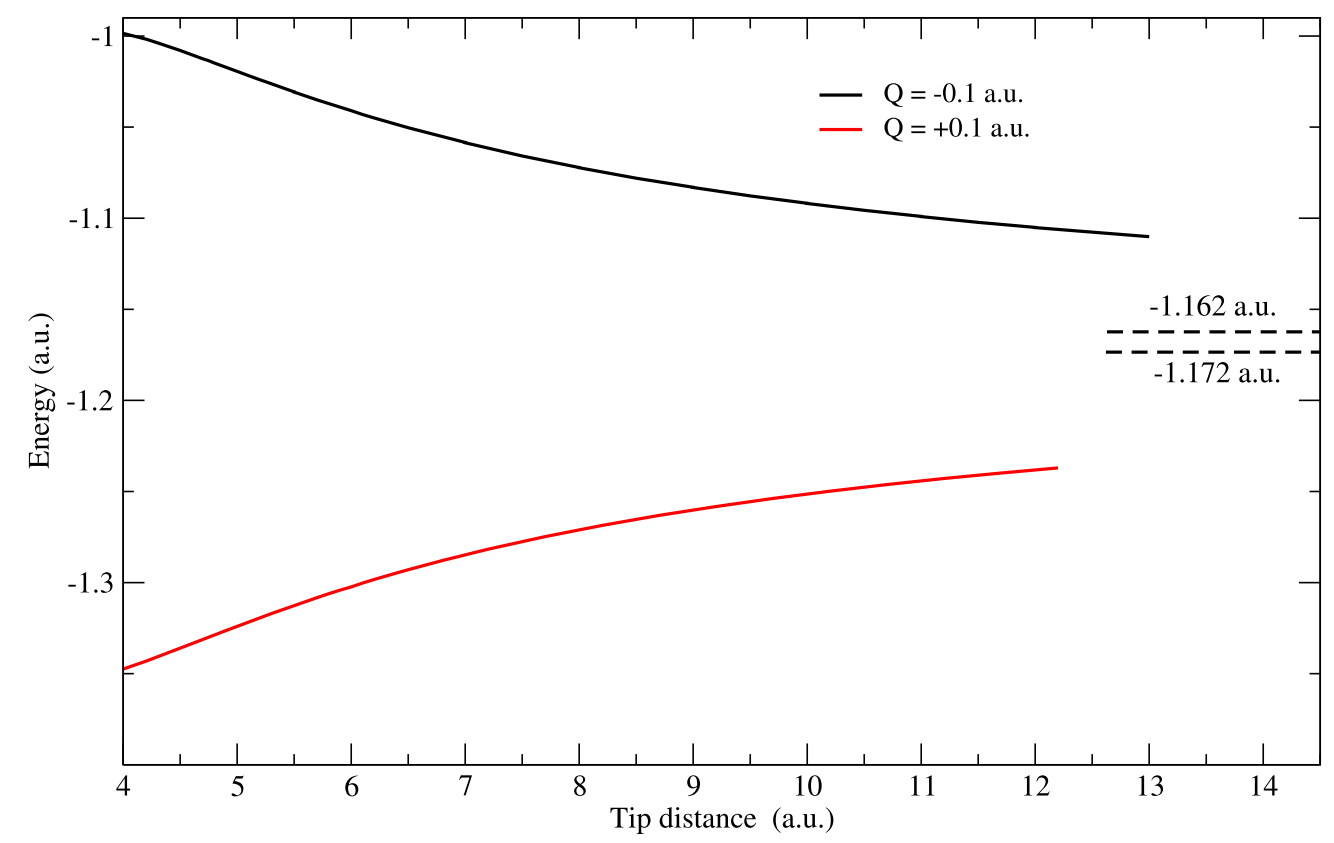

FIG. 2. Sampled adiabatic ground-state energy for PES with varying tip distance and both signs of a fixed amount of equal tip charge $Q=-0.1$ a.u., upper curve (black), and $Q=+0.1$ a.u., lower curve (red); the values obtained here for the free molecule, adiabatic (lower value) and nonadiabatic (upper value), are indicated at the right-hand side ordinate with dashed lines, which might be compared with $E=$ $-1.17448(2)$ a.u. of Tubman et al. [19]

\section{GROUND STATE}

In the BO approximation, we average Eq. (1) with the truncated wave function of Eq. (5) at static nuclear positions. This means that we leave out $\Phi_{\mathrm{nu}}^{(0)}$ and optimize the electronic variational parameters $\alpha_{k}$ for the potential energy surface (PES) $V^{\mathrm{PES}}(\mathbf{R})$. The latter is the expectation of the observable $\hat{H}$ without $\hat{T}_{\mathrm{nu}}$. The ground-state energy is obtained from the quantum-mechanical solution of the bare nuclear kinetic energy operator with $V^{\mathrm{PES}}(\mathbf{R})$.

Since our work focuses on the nonadiabatic treatment, we chose the Morse potential as an analytical approximation to $V^{\mathrm{PES}}(R)$ that is spherically symmetric and solvable in closed form. This eliminates the necessity of calculating an anisotropic PES in three-dimensional $\mathbf{R}$-space, and replaces it with the subsequent numerical integration of the isolated nuclear problem. The restriction to higher symmetry is used to efficiently compare with its respective nonadiabatic counterpart.

The resulting adiabatic energy is shown in Fig. 2 as a function of tip distance. The obtained asymptotic value of the free molecule is shown at the right-hand ordinate axis to be compared with that of Tubman et al. [19]. In the latter reference, the free case was treated to high accuracy with quantum Monte Carlo not restricted by the BO assumption. In the adiabatic part of our calculations, the molecule was aligned with the tip connection line, which reduces significantly the variational space for search of the energy minimum as a function of the nuclear positions. Please notice that, in the sense of adiabaticity, the nuclear positions are variational parameters. In fact, as will be seen in the following, the above alignment is totally lost for positive tip charge with not too distant tips. This adiabatic example leads to a caveat and its remedy consisting of the nonadiabatic free floating of the nuclear positions. The adiabatic approach itself is of course not to be blamed, since a restricted variational space might include a lower minimum if enlarged.

One could question parametrizing the nuclear positions only along the one-dimensional tip connection. The transfer of that dependence to its radial behavior in three-dimensional space will overstate the influence of the tips. Nevertheless, adiabatic procedures fix nuclear positions by premature optimization, which may occur within such inadequate regions and cannot be controlled in advance except by nonadiabatic testing.

Going beyond BO, the full wave function of Eqs. (5) and (6) was used for a minimum search of the ground-state energy. The analog of the adiabatic energy for the nonadiabatic case is presented in Fig. 3 by solid lines. Note that the ordinate scales in Figs. 2 and 3 differ by an order of magnitude. One observes that BO largely overestimates the energy difference between the positive and negative tip charges. In contrast, a strong stiffness against an external field is shown by the hydrogen covalent bond in the nonadiabatic case. Furthermore, for $Q>0$ a shallow minimum arises around a tip distance of 6 a.u. that falls below the large-distance level of the almost free molecule. A magnified view is shown in Fig. 4 with additional curves for a different value of positive tip charge. With increasing tip charge, the minimum deepens and shows an increase of the binding of the molecule center to the origin. It also proves the stability of the molecule's central position for positive tip charge under the imposed external electric field with respect to its free position far outside.

For negative charge, the attractive well becomes significantly deeper, a tendency that might correspond to a separate strengthening of the bonds between each atom and its closest 


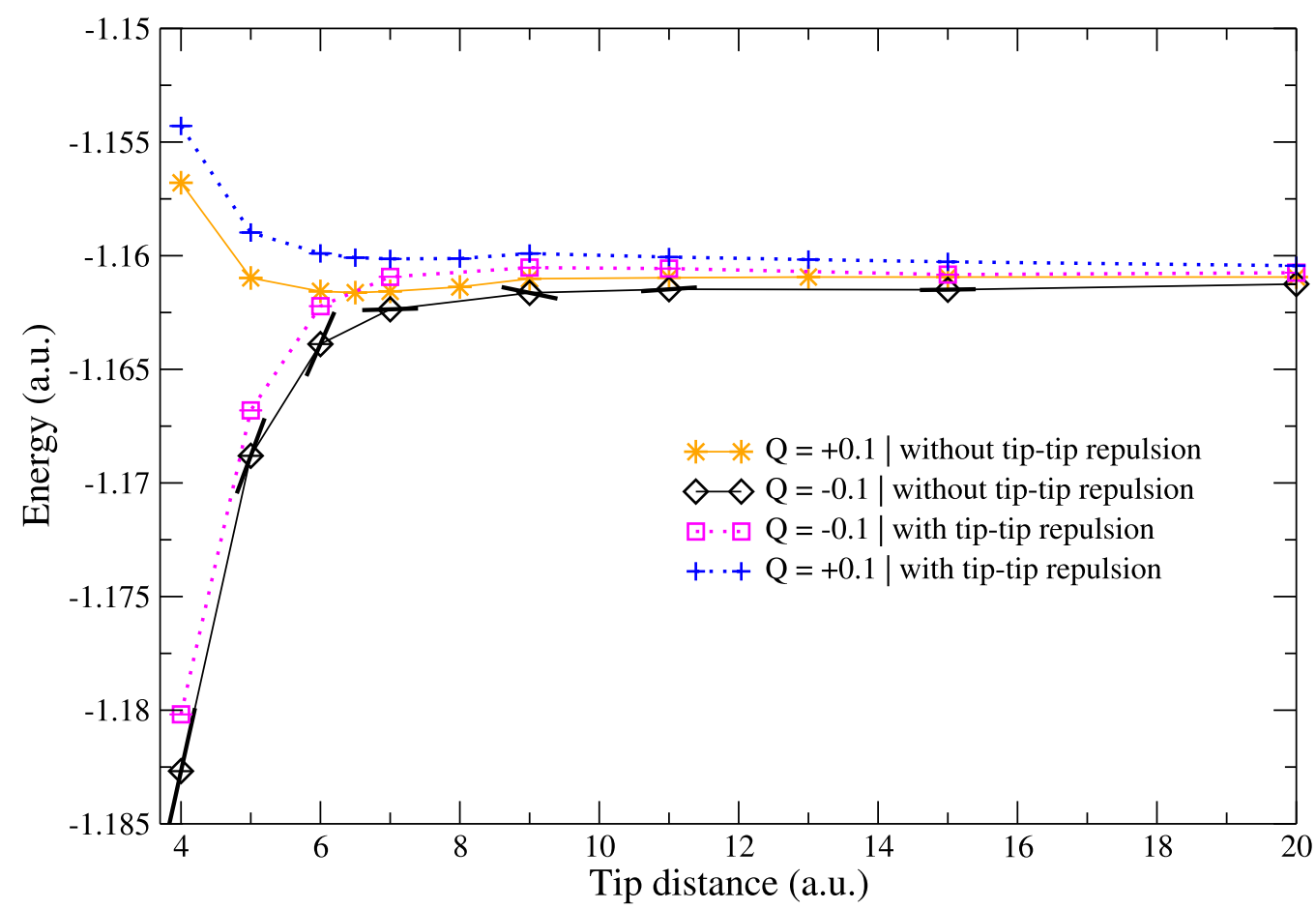

FIG. 3. Nonadiabatic ground states for axial symmetric nuclear wave-function ansatz plotted vs tip distance for both signs of tip charge: $Q=-0.1$ a.u. without tip-tip potential (diamonds, solid) and with tip-tip potential (squares, broken); $Q=+0.1$ a.u. without tip-tip potential (stars, solid) and with tip-tip potential (pluses, broken). Straight line sections at negative tip charge points represent tangents at those distances obtained from the virial equation; see, e.g., [21].

tip neighbor in a kind of physisorption. We calculated the force in order to check the accuracy of the plotted data and obtained the tangents at the chosen points as shown in Fig. 3. The derivative with respect to tip distance $D$, $\frac{d E}{d D}=-\frac{1}{D}\langle 2 T+V\rangle$, follows from homogeneity by a scaling argument as usual [21]. By adding the electrostatic tip-tip

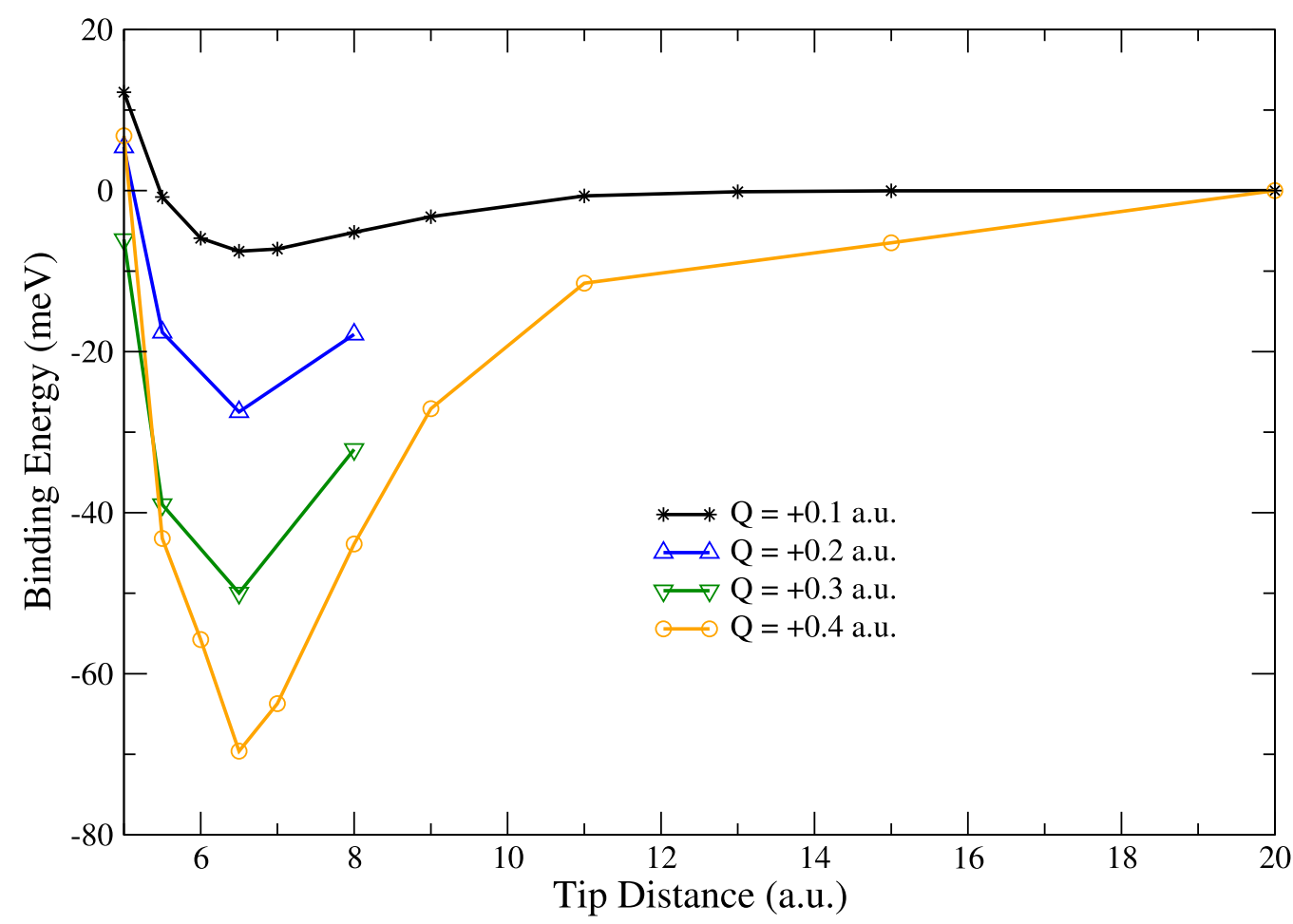

FIG. 4. Ground-state energy vs tip distance for equal positive tip charge of various amounts $Q=0.1,0.2,0.3$, and 0.4 a.u. plotted by stars, up triangles, down triangles, and circles, respectively. 

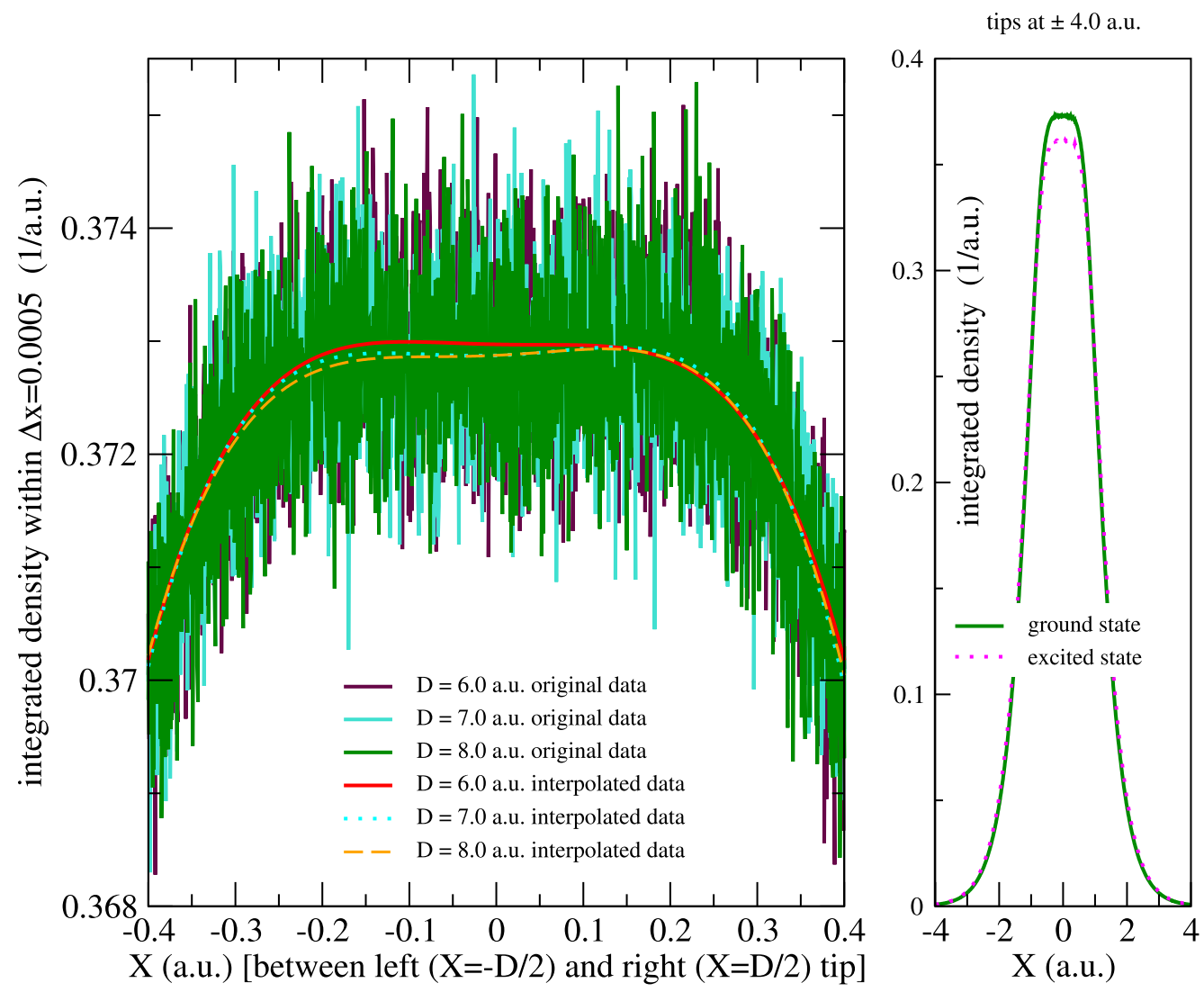

FIG. 5. Electron density (per electron) integrated over $Y Z$-sheets of $\Delta X=0.0005$ a.u. thickness. Left: section around the maximum of original data plotted by originally scattered and smoothed lines, respectively, for tip distances of $D=6,7$, and 8 a.u. and a tip charge of $Q=$ +0.1 a.u. for the ground state of the nuclear wave-function ansatz of spherical symmetry. Right: extended curve for $D=8.0$ a.u. with both ground (full) and first vibrational excited (dotted) state.

repulsion potential (see the dashed lines in Fig. 3), one observes that the complete resulting potential between the tips proves to be overall repulsive for positive charge, but it remains attractive for negative charge. The electrostatic tip-tip interaction is rather smooth and small compared to the drastic tip-molecule dependence on the charge's sign and size.

Including the electrostatic tip repulsion describes a dynamical system similar to an atomic force microscope (AFM) with $\mathrm{H}_{2}$ between tip and surface. The shallow energy minimum apparent for positive charge and indicating stability of the molecular configuration within clamped tips vanishes due to the strong electrostatic repulsion if the tips are left free to move, which then occurs in opposite directions. In the case of negative charge, the tip repulsion cannot dominate over the attraction by the molecule's protons, i.e., it cannot balance the intramolecule attraction over the whole system. The tips will approach, at least in the region of displayed distances in Fig. 3. At closer approach it can be questioned whether the molecule remains aligned and the bond eventually opposes a further tip approach or turns to a perpendicular direction with decreasing tip-tip distance, thereby decreasing the proton-tip attraction. This is discussed later in this paper. Also, we recall that the nuclear center of mass is fixed to the origin.

We show in Fig. 5 a plot of the electronic density for a few distances of the tips. The sampled density is collected in small sheets perpendicular to the cylindrical $(X)$ axis. The nuclear wave-function ansatz in this example has purely spher- ically symmetric parts and a one-dimensional radial nuclear sampling is used, neglecting angular contributions from the nuclear coordinates' presence in the electronic wave function. Apparently, the electron cloud is arranged over a disk of average extension equal to the hydrogen bond length along the $x$ axis. Small fluctuations and a close resemblance of the curves are observed in the range of the few tip distances shown, i.e., a rather inert chemical bond exists at this level of accuracy. The plateau at maximum electron density shows two small elevations indicating the $\mathrm{H}_{2}$ nuclear sites' double structure but no visible influence from the varying distance of the tips.

The probability density of the nuclear positions sampled during a Monte Carlo run illustrates what happens to the molecular axis for different amounts of tip charge, for central and axial symmetry, and when approaching the tips. Being a quantum-mechanical observable, the orientation of the molecular axis fluctuates. It may be expected to change if external parameters are changed. The hydrogen-axis direction is recorded by the distribution of its polar angle to each of the three Cartesian axes $X, Y$, and $Z$. In Fig. 6 the probability density $\rho(\theta)$ for a polar angle $\theta$ is plotted for the case of a spherically symmetric nuclear wave function of an isolated $\mathrm{H}_{2}$ molecule $(Q=0)$, which we still allowed to have full three-dimensional degrees of freedom in the sampling. The directional fluctuations from a homogeneous distribution of $\frac{1}{2} \sin (\theta)$ are small, as is illustrated by a magnified plot section in the inset. 


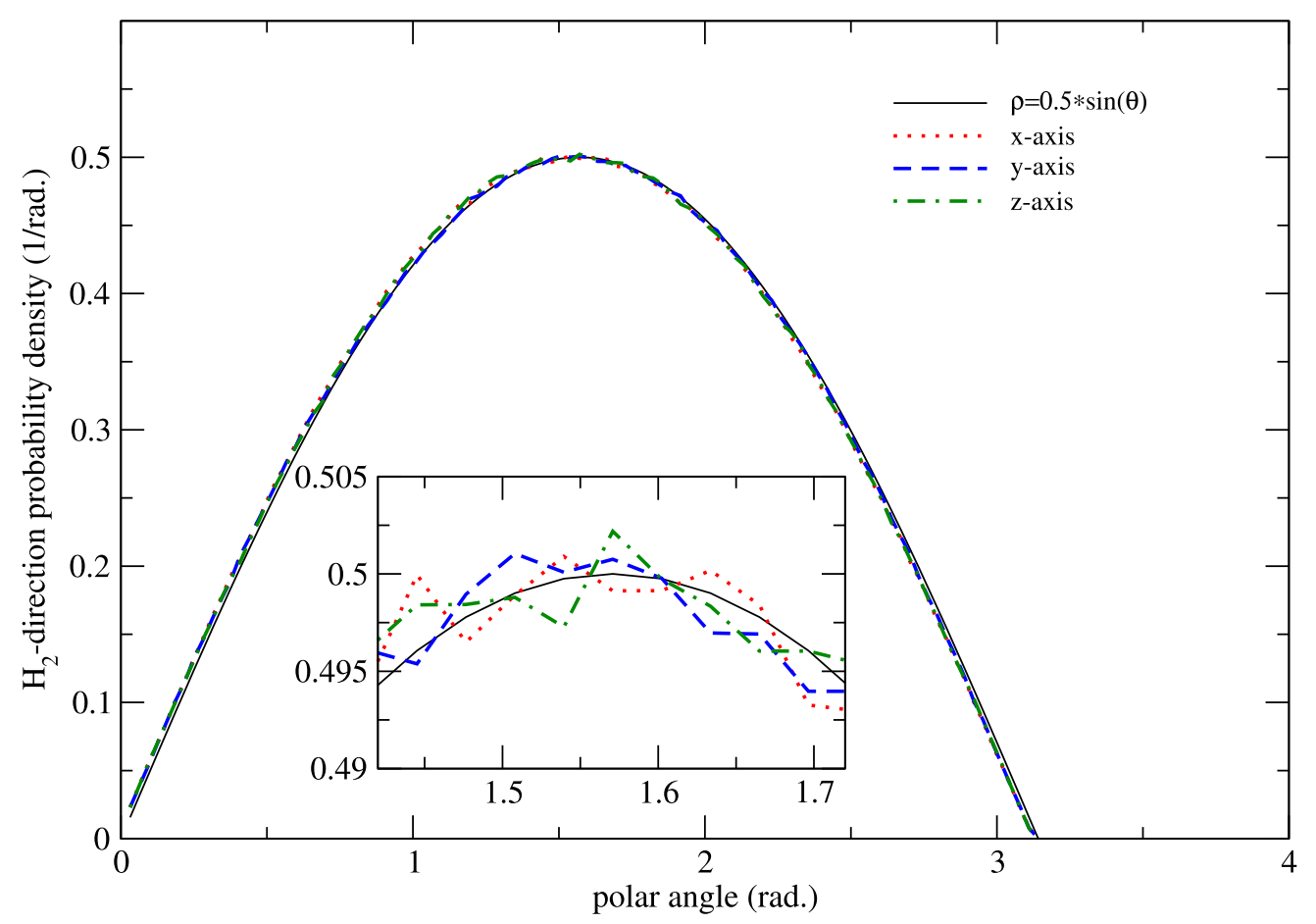

FIG. 6. Isolated $(Q=0) \mathrm{H}_{2}$ molecule: Probability density of polar angles of $\mathrm{H}_{2}$ axis with respect to $X, Y$, and $Z$ axes sampled in full three-dimensional space; amplified resolution shown by the inset.

In contrast, with finite tip charge a drastic change is observed when the positively charged tips are approached: A symmetry breaking increasingly takes place toward axial symmetry. The electrostatic repulsion between tips and partly unscreened nuclei turns the molecular axis distribution toward the equatorial plane. The turning of the $\mathrm{H}_{2}$ axis follows qualitatively this electrostatic picture according to the tips' field distribution. This illustrative explanation needs a strong electronic screening of the proton charge to reduce the electrostatic energy by an order of magnitude to the QMC computed quantum-mechanical value. The emergent turning of the molecular axis is illustrated by Fig. 7, where both cases of tipcharge sign are displayed. The presentation is slightly different from that in Fig. 6 with the reference polar axis fixed along the tip-tip connection line ( $X$ axis). The abscissa here denotes the polar angle $\theta$ with respect to the $X$ axis. A few values of the azimuth angle $\phi$ appear as a plot parameter. The $\theta$ accumulation around $\pi / 2$ for positive tip charge indicates a preferred direction near the equatorial plane. The two-lobe distribution then corresponds to the two opposite cones into which the internuclear distance vector can point in the case of negative tip charge. The azimuth distribution is apparently uniform, as it should be. The slight asymmetry toward a more frequent pointing to the positive hemisphere originates from the initial start position of the sampling. Note the implicit sine-factor weight from the azimuthal degree of freedom present in the distribution that forces the distribution to zero at both borders.

Aside from the very pronounced depression in the case of negative tip charge, where a large attraction after an initial repulsion is seen for decreasing tip distance (see Fig. 3), a barely noticeable small dip appears in the ground-state energy of positive tip charge between large and small tip separation. The amount of the depression, however, increases for larger tip charge and could be resolved by an AFM. We investigated larger positive tip charges. The results are shown in Fig. 4. The shapes of the curves together with the position of their depression minima are rather independent of charge. The depth appears to increase rather linearly with tip charge such that energies in the range of a few hundred $\mathrm{meV}$ are attainable. We thus observe already in this simple system a trapping of the molecule that has been stated by Wang et al. [7] for nanocavities built by an atomic environment at a surface. Additionally, the trapped $\mathrm{H}_{2}$ in this potential well, where the tip distance can be mapped onto the tip bias, experiences a bias-dependent varying force. By this nonlinearity, the $\mathrm{H}_{2}$ may couple to an external oscillator connected with the tip, as realized in an elegant design [8] for a $\mathrm{H}_{2}$-covered $\mathrm{Cu}(111)$ surface.

In other words, for both signs of charge there exist regions of total energy below its value for the free molecule. The system will prefer those parameter regions with energy below the vacuum level and, in addition, highest symmetry. The center of mass possesses these properties at the origin. As the term "stability" in nuclear position space refers primarily to an adiabatic description, we use it only loosely in this nonadiabatic discussion. In preliminary samplings of the center of mass, we found that its quantum-mechanical distribution peaked at the origin and monotonously but slowly decreased toward the sides. Of course, since we do not perform global optimization, a better result may still exist.

\section{VIBRATIONAL EXCITATION}

\section{A. Concept of nuclear wave function}

In this paper, we consider the molecular vibrations as key to measurement. Variational QMC schemes are primarily 


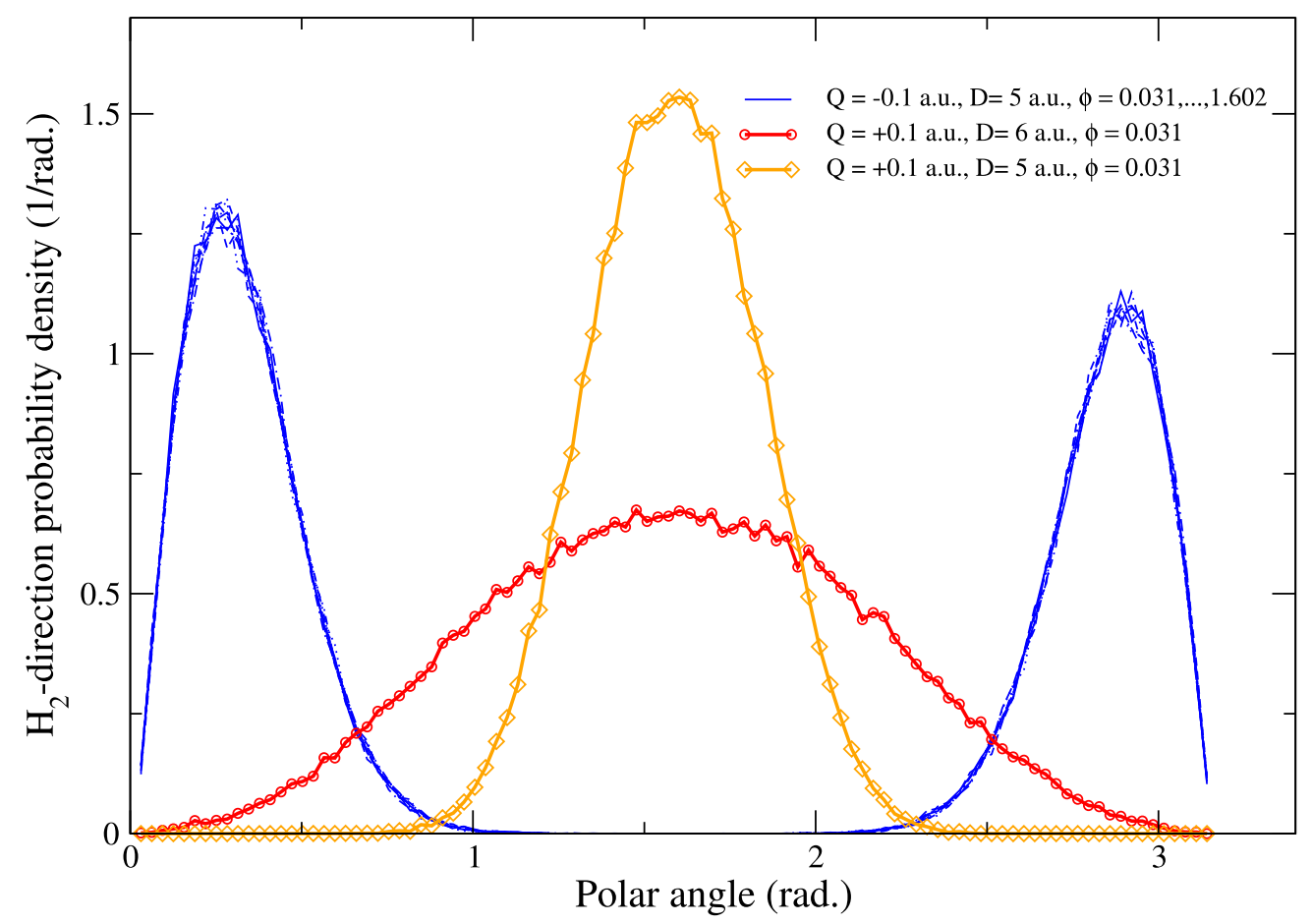

FIG. 7. $\mathrm{H}_{2}$ molecule embedded between tips $(Q \neq 0)$ : Equatorial and conical probability distributions of molecular axis vs polar angle in the strong axial symmetry regime of the ground state: tip charge $Q=-0.1$ a.u. with thin solid and dashed lines (blue) for a set of azimuths $\phi=0.031,0.063,0.094,0.126,1.508,1.539,1.571$, and $1.602 \mathrm{rad}$ for tip distance $D=5.0$ a.u.; tip charge $Q=+0.1$ a.u. for azimuth $\phi=$ $0.031 \mathrm{rad}$ with solid line (orange, open diamond) for $D=5.0$ a.u. and solid line (red, open circles) for $D=6.0$ a.u.

devoted to ground-state investigations [21]. However, the conceptual simplicity of the nuclear wave function reduces the variational efforts for excited states by providing near orthogonality through symmetry arguments. As the ground state has the highest symmetry, we choose, similar to the harmonic case, an ansatz that is antisymmetric in the stretching mode coordinate for the first vibration amplitude. This ansatz is motivated by assuming a smooth transition from the free molecule with spherical symmetry to its constrained symmetry with one remaining rotation axis, and the choice of a spheroid suggests itself from the ground- and excited-state exponent's elliptic expression as a variational ansatz. In this way, cylindrical symmetry of the whole system is obeyed. Nevertheless, it complicates the notion of vibrational modes of free binary molecules. In fact, if we refer to the bridging experiments and focus on the stretching mode, a purely radial eigenmode does not exist in cylindrical symmetry. Instead, aside from the rotational eigenmodes around the tip-tip axis, the other modes generally contain a mixture of a stretching vibration and a rotation with polar angle referring to the tip-tip axis. Besides, the concept of a stretching mode now corresponds in the quantum-mechanical continuous space description to a breathing mode in purely spherical symmetry with additional distortions by differential rotation in axial symmetry.

The surface of the wave-function zero is adapted from the free molecule's spherical shape to a spheroid as a function of the nuclear relative coordinate $\mathbf{R}=\mathbf{R}_{\mathbf{2}}-\mathbf{R}_{\mathbf{1}}$. This assumption complies with the axial symmetry and a perpendicular mirror. It is further specified by adjusting a simple quadratic polynomial to the expected linear behavior in the neighbor- hood of the zero at the spheroid. Two cases are encountered depending on the ground-state behavior: the prolate spheroid may be elongated along the tip-tip direction, as, e.g., for negative tip charge, while the oblate spheroid is flattened perpendicular to that direction for positive charge. From now on, we focus on the former case as it possesses a large stability region, and we write

$$
\begin{aligned}
\Phi_{\mathrm{nu}}^{(1)} & \left(\mathbf{R}_{1}, \mathbf{R}_{2}\right) \\
= & \left(X^{2}+\frac{Y^{2}+Z^{2}}{1-e^{2}}-a^{2}\right) \exp \left[-\zeta_{x}^{(1)}\left(X-R_{x}^{(1)}\right)^{2}\right. \\
& \left.-\zeta_{\rho}^{(1)}\left(\sqrt{Y^{2}+Z^{2}}-R_{\rho}^{(1)}\right)^{2}-\zeta^{(1)}\left(|\mathbf{R}|-R^{(1)}\right)^{2}\right]
\end{aligned}
$$

as the nuclear factor of the vibrational excited wave function. The spheroid is characterized by its semimajor axis $a$ and numerical eccentricity $e$ as variational parameters together with $R^{(1)}, R_{x}^{(1)}, R_{\rho}^{(1)}$ for the variational centers and $\zeta^{(1)}, \zeta_{x}^{(1)}, \zeta_{\rho}^{(1)}$ for the variational widths of the Gaussian ansatz. The oblate case would instead have the factor $\frac{1}{1-e^{2}}$ multiplying the term $X^{2}$. Orthogonality to the ground state is imposed by integrating the mutual projection $\left(\Phi_{\mathrm{nu}}^{(1)}, \Phi_{\mathrm{nu}}^{(0)}\right)$ of the respective nuclear factors and choosing the semimajor axis value to set this scalar product to zero.

\section{B. Polarization of vibrational modes}

The eccentricity can be treated as a parameter either free for energy minimization or prescribed by assuming the vibration polarization in advance for some special cases. The latter are considered here as follows: by using a sphere with $e=$ 0 for a quantum-mechanical breathing mode corresponding 


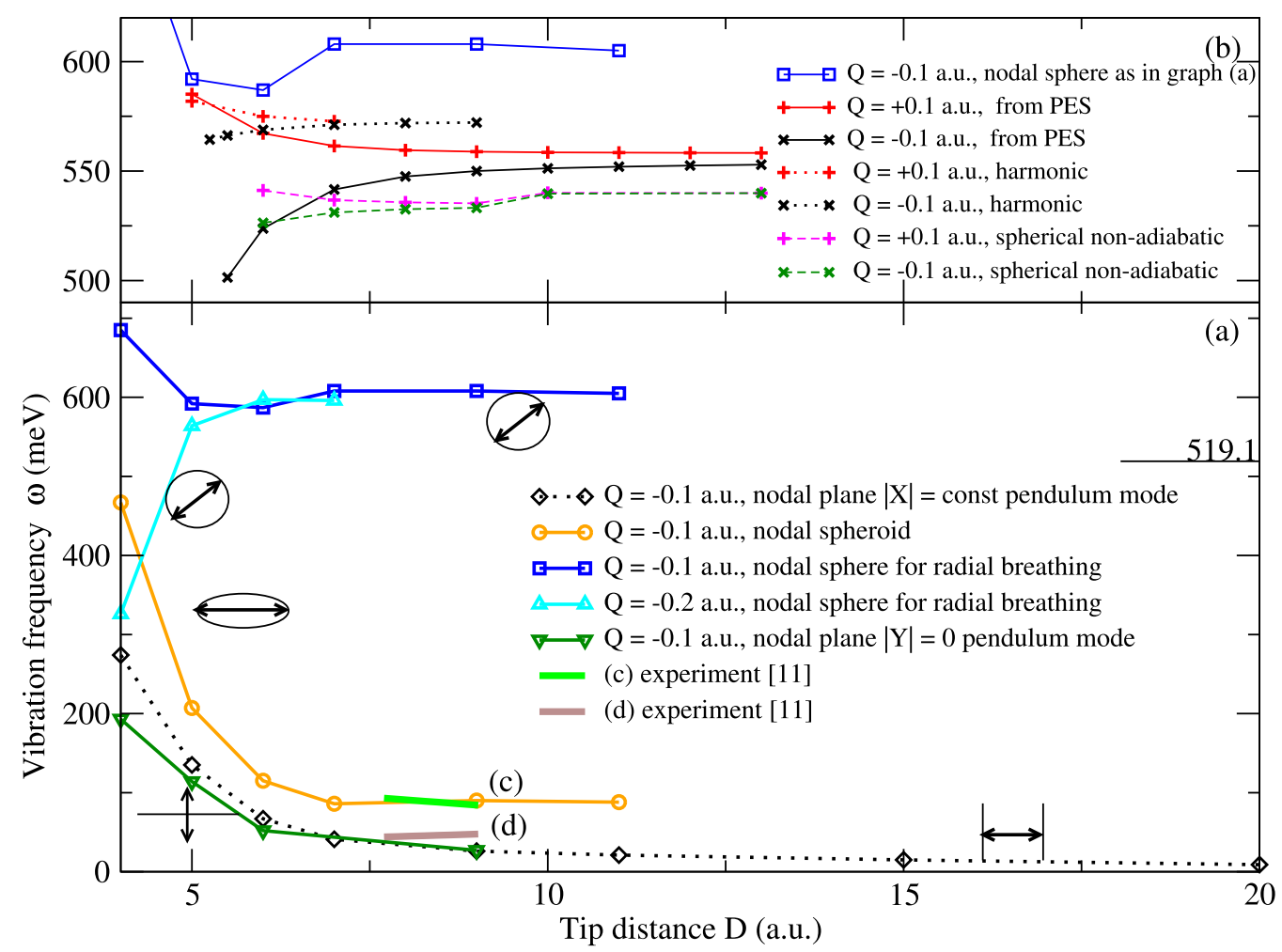

FIG. 8. Lowest vibration excitation frequencies vs tip distance for various vibration modes and approximations. Graph (a) (nonadiabatic vibration modes): pure breathing mode (squares, solid, blue) with spherical nodal surface, pendulum mode (circles, solid, orange) with optimized spheroidal nodal surface, pendulum mode (down triangles, solid, green) with node on plane $Y=0$, and pendulum mode (diamonds, dashed, black) with node on plane $|X|=$ const all for tip charge $Q=-0.1$ a.u., and the pure breathing mode (up triangles, solid, cyan) for tip charge $Q=-0.2$ a.u. with spherical nodal surface. Literature value [16] of the free molecule is marked at the right ordinate. Icons with arrows near a curve symbolize vibration polarization mode by its shape of the nodal surface projection onto the $(X, Y)$-plane as circles and ellipses, the latter also with its degenerate linear shapes $Y=0$ and $X= \pm X^{(1)}$. Experimental curves [11] of Fig. 3 in that reference, right-shifted by 9 a.u., are depicted by two almost straight lines labeled as (c) and (d). Graph (b) (adiabatic to nonadiabatic comparison): a section of the pure breathing mode (squares, solid, blue) is repeated from (a) to compare with three less accurate results obtained from one-dimensional sampling of the radial nuclear distance coordinate, i.e., assuming full spherical symmetry, (i) on the basis of BO with PES by fitting to a Morse potential after QMC electronic parameter optimization for tip charge $Q=+0.1$ a.u. (pluses, solid, red) and $Q=-0.1$ a.u. (crosses, solid, black), (ii) on the basis of the harmonic approximation (dotted, red/black) for both charges $Q=+/-0.1$ a.u., respectively, and (iii) on the basis of a nonadiabatic treatment in this forced full spherical symmetry $Q=+0.1$ a.u. (pluses, dashed, magenta) and $Q=-0.1$ a.u. (crosses, dashed, green).

to the classical stretching mode; or a plane with $e=1$ and node $Y=0$ for a rovibrational mode of pendulum type in the $(X, Y)$-plane; or a twofold plane $|X|=X^{(1)}$ of pendulum type as well, but with azimuth symmetry around the tip-tip axis. By the specific choices of the nodal surface we introduce a confinement of the nuclear Hilbert space that restricts its low-lying rovibrational excitations. We expect them to play a role in the inelastic electron transfer of the hydrogen bridge experiment as transition states weighed by respective matrix elements.

The local velocity at and close to this nodal surface is directed normal to the surface along the intersecting confocal hyperbolas within any plane containing the tip-tip axis. It is composed of a radial stretching vibration and a rotational component that increases with increasing eccentricity $e>0$. The additional rotation is due to the tips' symmetry breaking with an energy amount corresponding to the tip charge, roughly speaking. Thus, the degree of freedom connected with the polar angle mixes into the stretching vibration eigenmode.
As the low rotational mode frequencies are smaller by an order of magnitude, one could expect the mixing to only slightly decrease the vibration frequency for small perturbations by the tips' field. Altogether the field becomes strong for very small tip distance, a regime where the hydrogen bond already starts to break. In that regime, the field appreciably pulls down the vibration frequency anyway, as seen in Fig. 8; this behavior arises as a general feature when compared with various assumptions about wave-function approximations presented in part (b) of that figure.

Considering the spheroidal nodal surface in the case of negative tip charge, we expect from the ground-state wavefunction shape a prolate spheroid along the tips' connection line. The three limiting cases of eccentricity $e=1$ occur with the planar degenerates of an ellipsoid, i.e., two planes $Y=0$ and $Z=0$ containing the $X$-axis with breaking of the ground state's axial rotation symmetry, and a rotationally invariant plane perpendicular to the $X$-axis consisting of two symmetrical parts at $X= \pm X_{1}$. The former two states have 


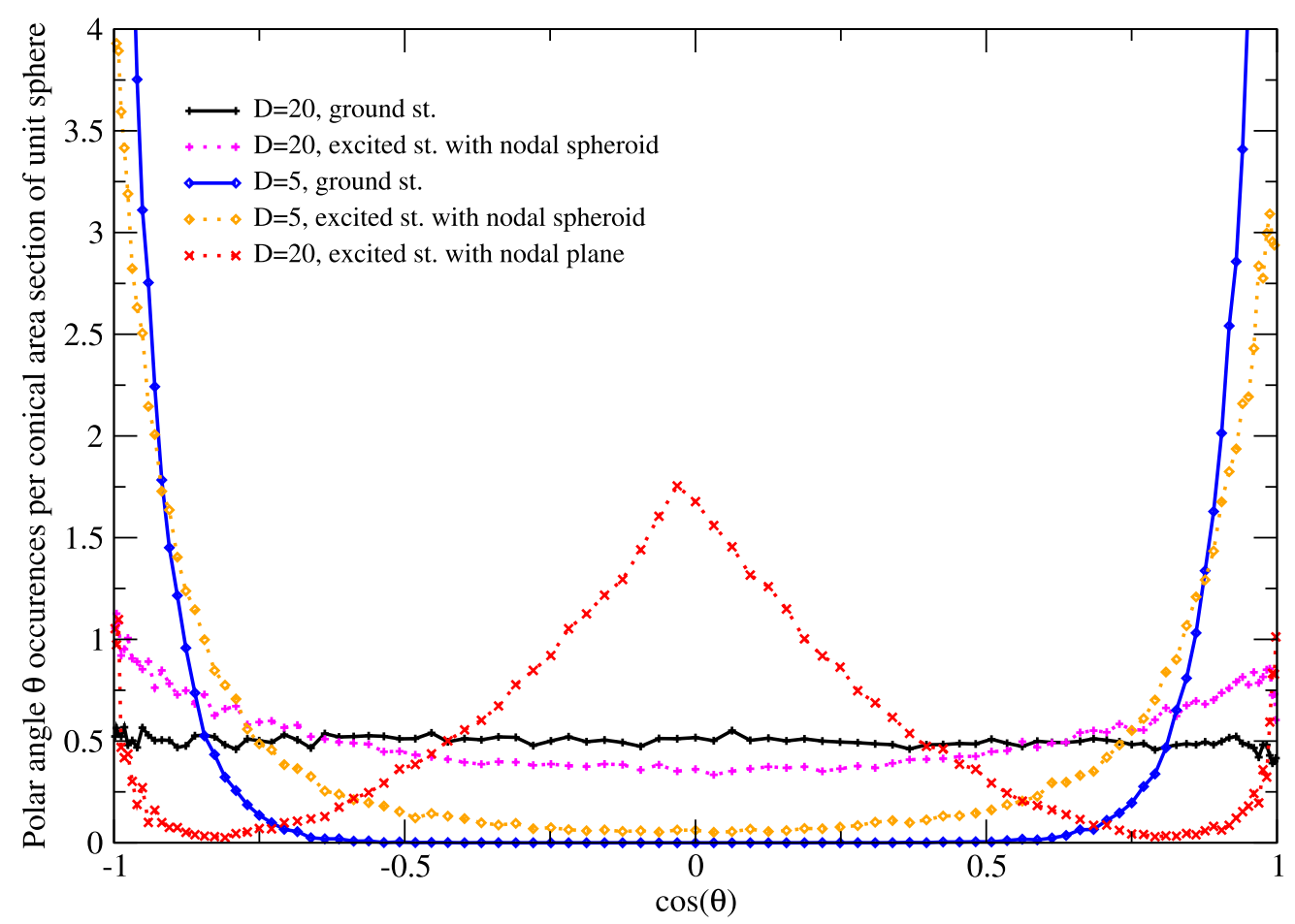

FIG. 9. Normalized occurrences of polar angle between tip connection and hydrogen axis vs cosine of polar angle $\theta$. Occurrences refer to the ring area on the unit sphere between $\theta$ and $\theta+d \theta$ : ground state (solid) for tip distance $D=20$ (pluses, black) and for $D=5$ a.u. (diamonds, blue), vibrational excited state for stretching mode on a spheroidal nodal surface (dashed) with $D=$ 20 (pluses, magenta) and 5 a.u. (diamonds, orange), and rotation-vibration excited state with nodal plane at $|X|=$ const for $D=$ 20 a.u. (dashed, crosses, red).

definite rotation axes like a pendulum mode with the molecule axis swinging across the pole around the $Z$-axis with node $Y=0$ and its equivalent with node $Z=0$. These vibrational states will be denoted here by $\Pi_{Y}$ and $\Pi_{Z}$, respectively. The last case, which we denote by $\Pi_{Y Z}$, resembles a classical stretching vibration along the tip-tip axis, but it corresponds quantum mechanically to a breathing mixed with a sliding of the probability distribution along this axis.

\section{Transition probability}

The variational states that we obtained are eigenstates, i.e., long-living, within our accepted range of accuracy given by a square-root value around 0.05 a.u. of local energy variance and around 0.000005 a.u. of total energy expectation variance. Calculating the inelastic electron transfer observed in experiment must determine an acceptable excited state by the transition matrix element aside from its ground-state orthogonality. As a rough estimate, we consider in addition to symmetry selection rules the directional overlap of the excited state with the ground state including an additional one-electron orbital on both tips being occupied, say $\phi_{R}$ on the right-hand side in the ground state $\Phi^{(0)}$ and $\phi_{L}$ on the left-hand side in the excited state $\Phi^{(1)}$. For example, a transfer matrix element $\left(\phi_{L} \Phi^{(1)}, V_{D} \phi_{R} \Phi^{(0)}\right)$ has to be investigated for hydrogen-assisted or hydrogen-blocked inelastic tunneling using the tunneling theory [22], STM theory [23], or a scattering $t$-matrix [24] for impurity-assisted tunneling processes. The driving potential for an electron transfer $V_{D}$ consists of a voltage drop between the tips illustrated, e.g., by an additional static negative point charge on the right tip. It also has rotation symmetry with respect to the $X$-axis, thus allowing, e.g., also for the $\Pi_{Y Z}$-type state described above at least by symmetry.

Examples for two tip distances are displayed in Fig. 9 showing the similarity in the axes' angle distributions for the ground and excited states. Such a similarity increases the transition probability from the ground state to the excited state and would favor an inelastic electron transfer via a hydrogen bridge, provided that the operator in the matrix element depends only smoothly on the angle. Conversely, the $|X|=$ const planar nodal surface has less overlap with the ground state at a tip distance of 20 a.u., and, additionally, it shows this angular overlap around the equatorial plane, which the orbital of a tip electron rarely could access in order to tunnel. These qualitative arguments do not strictly exclude other parts from the large excited states' space of the rotation-vibration spectrum even at the low excitation level of some hundreds of meV. Even the excited state with a planar nodal surface, which has been ruled out above, shows similarity with the bridge experiment insofar as its energy is monotonously decreasing with increasing tip distance. The leading selection considered here is based on the orthogonality to the ground state and the continuity with the isolated molecule. A definite answer would need a calculation of the transition matrix element and is beyond the scope of this work.

\section{Vibration frequencies}

Before discussing the final vibration frequencies resulting from our calculations, we show in part (b) of Fig. 8 the frequency dependence on the tip distance for three approximate schemes, namely a harmonic approximation to the sampled 
BO PES, a Morse potential fit to the PES itself, and a nonadiabatic calculation with the assumption of spherical symmetry of the nuclear wave function. The vibration frequency softens (hardens) for negative (positive) tip charge with increasing external field strength due to approaching tips. All those results based on spherical symmetry are not influenced by the turning molecule axis that has been found in the ground-state calculations as a rotation into the equatorial plane for positive charge and as an aligning with the tips for negative charge. Such differences in configuration are expected to affect the vibrational modes and the process of their excitation.

In fact, the actual axial symmetry forces a hybridization of vibration and rotation of an eigenstate. For example, the transition from the free molecule with a radially symmetric nuclear wave function to the molecule bond by the tips in cylindrical symmetry gradually restricts the molecule's motion within some cone around the tip connection line or within an equatorial disk depending on charge.

Figure 8 helps, by means of different ordinate scales of parts (a) and (b), to judge the magnitude of change introduced by a nonadiabatic treatment. Although the shapes of the respective curves look similar, their direction and amount of shift show clear differences and seem difficult to estimate in advance.

The difference between the excited state and the ground state yields the vibration energy plotted in Fig. 8. A detailed description of the ground- and excited-state curves themselves may be found in the Supplemental Material [25]. The spheroidal eigenmode is shown to soften appreciably with decreasing tip distance $D$. It approaches with increasing $D$ both the $\Pi_{Z}$ pendulum mode and the limiting $\Pi_{Y Z}$ rotationvibration mode, which themselves approach each other still closer. The spherical mode shows a slight bending downward with decreasing $D$ from large values for $Q=-0.1$ a.u. and a much stronger bending for $Q=-0.2$ a.u. with an onset already at higher $D$. A similar behavior shows the stretching mode of the DFT simulation of Djukic et al. [11] in its high-energy portion with $D>4$ a.u. if $Q$ is adjusted here to somewhat larger negative charges. The curves we find for the pendulum and the rotation-vibration modes in Fig. 8 have a shape that could with some imagination be associated with the tail of the transverse pendulum mode. Here, we identify them by a purely lateral motion along the $Y$-axis or longitudinal motion along the $X$-axis with a fixed center of mass. Thus, the association of our curves with density functional theory (DFT) calculations and experiment presents some ambiguity because of the notion of polarization in terms of classical or quantum mechanics on the one hand, and because of the different tip distance ranges used on the other hand.

The QMC calculations may also be directly compared with the experiment, although the measurement does not specify a polarization signature of the wave function with respect to the vibration eigenstate. One can try to associate in that case the shape of curves with specific excited states from QMC, namely a spherical breathing mode and a spheroidal mode where breathing is mixed with shearing. Because of its high excitation energy, the breathing mode we obtain here is not accessed by experiment. Our curve with doubled tip charge $Q=-0.2$ a.u. tends to lower the energy, which, however, occurs at too small a tip distance. We do not know what the zero of the experimental abscissa is, so we feel free to adjust it yielding the curves denoted (c) and (d) in Fig. 8(a). This shall not suggest an approximation to experiment as our external parameters $(D, Q)$ would have to be realistically mapped onto the measured situation. But it gives an impression of which portion of the tip distance might correspond to the rather small measured region. The value of the frequency as well as the slopes of the curves suggest that tip distances above 7 a.u. can probably be associated to our spheroidal mode with one of the curves $a$ or $b$.

The cited DFT calculations-being supported by our own calculations on a linear chain of $\mathrm{Au}$ atoms with a gap bridged by a hydrogen molecule-yield in addition the wave function's fingerprint as being longitudinal or transverse and with the center of mass being fixed or oscillating. Our DFT results indicate that the $\mathrm{H}_{2}$ bond breaks below 10 a.u., which compares with 7 a.u. from QMC where the hydrogen bond starts to weaken significantly. At this distance, the main $s$ orbital broadens appreciably with a corresponding bond-length increase. Thus, there is agreement between DFT stretching and QMC breathing modes. The remaining pendulum mode is rather unspecific and not easily identified. At least it does not contradict the pendulum mode of DFT. The difference could originate from the use of the $\mathrm{BO}$ approximation in DFT as opposed to the QMC nonadiabatic treatment with a basically different concept of correlation. But there could also be a simpler reason buried in the correspondence between both models, i.e., between the external parameters $D, Q$ of the QMC bridge model with a pointlike tip charge and tip distance and the DFT model with atomically structured tips.

The findings in the bridge [11] and cavity experiments [8,9] for the vibration energy versus tip distance curves show some similarity to the data presented here. Two experimental curves of Djukic et al. [11] deserve special consideration, as they are in the reach of the parameter space covered here; see Fig. 8.

\section{CONCLUSIONS}

For the geometry of the system that we have studied here, QMC calculations have been performed to obtain the total ground-state energy for both signs of charge. The results show overall stability for a negative tip charge. A charge of equal but positive amount yields stability with respect to the free molecule for tip distances larger than around $D=5$ a.u. The deviation from the $\mathrm{BO}$ evaluation can be estimated by the energy difference of both charge signs between the PES and the nonadiabatic case, which amounts to 0.3 a.u. at about $D=10$ a.u. The quantum-mechanical concept of the protons' wave function significantly reduces the differences appearing in a classical treatment within the BO frame. In addition, the molecular orientation adjusts itself to an external field yielding in addition to the strong reduction of the energetic difference also an unexpected qualitative difference in the proton-proton angular wave-function distribution. The probability distribution of molecular orientation in space is important for the molecule's reactivity with a neighboring molecule. It can be influenced by the tip separation and switched by the tip charges into an a priori preferred direction. A technical byproduct of the present work proved to be the necessity of some 
repetitions in optimizing in turn the variational parameters of the nucleon-only part and those of the electronic wave function part, which is beyond a variational BO decoupling. Total energies could already be improved by around $100 \mathrm{meV}$ by the second optimization of the electronic parameters after the nuclear parameters.

While our discussion focused on a two-tip/one-molecule system, it might help the next step toward more complicated and extended nanomechanical objects as an example for an incorporated hydrogen molecule with electrically induced switching properties.

The molecule can be examined by detecting its vibrational spectrum. The results for the first vibrational excitation show that the energy can be resolved by QMC. Qualitatively a similarity emerges between the full quantum-mechanical view and a purely electrostatic view of the proton dynamics for suitably screened charges. The quantitative results for the free molecule are determined with high statistical accuracy (usually linewidths in the plots) of the QMC scheme. The systematic error can be asymptotically controlled by reference to the free molecule value. The absolute value of the ground-state energy is estimated to lie about $60 \mathrm{meV}(\sim 10 \%)$ above the exact value for the free case, whereas the vibration frequency is found to deviate by about $70 \mathrm{meV}(\sim \pm 10 \%)$ relative to the values of the free molecule.

A variety of curves of similar shape are shown in the plots of Fig. 8 of vibration frequency versus tip-tip distance for different approximations and for different polarization modes. They reveal appreciable differences between the nonadiabatic treatment and the adiabatic approximation. A comparison with the bridge experiment requires a discussion of the transition matrix element to specify the involved polarization of the vibrations. The polarization depends on the symmetry of the initial and final state including the associated orbitals of the tunneling electron. The experimental vibration modes find a theoretical counterpart as a $\Pi_{Y Z}$ mode and a degenerate pair of $\Pi_{Y}$ and $\Pi_{Z}$ modes. Further support in the discussion of the transition matrix is obtained from the directional overlap of the nonadiabatic wave function of the $\mathrm{H}_{2}$ molecule with the orbitals of the tunneling electron.

In any experimental realization, equal positive charges on both tips designed as above work similarly to an anode, whereas negative test charges could be offered to the molecule by one or more additional tips in the mirror plane as a cathode. Parts of such a charge can be thought to propagate via the molecule from cathode to anode. Also, equal positive charges on both tips direct the hydrogen molecule axis mainly transverse to the tip-tip line, where it is uniformly distributed over the azimuthal angle. Then the distance between the tips and the hydrogen electronic charge cloud, even if somewhat polarized and constricted in its lateral dimension, is larger than it is in the case of no tip charge at all, which corresponds to a spherically symmetric hydrogen charge cloud. And that additional distance will be still more pronounced compared with the case of negatively charged tips with an elongated distribution toward the tips. Thus, changing the bias or the distance will open a channel that, with suitable additional driving voltage, may exchange electrons between the tips through the hydrogen bridge: less in the case of positive tips and more in the case of negative tips.
In terms of surface physics, the validity of our calculations with respect to effects considered here is limited to the field of physisorption, i.e., the $\mathrm{H}_{2}$ molecule should not be dominated by covalent bonds to the tips. Including chemisorption to the tips would not present a difficult computational obstacle, as one could add bonding orbitals centered on the tips. Starting at the optimization level obtained here, one would need a similar number of additional computer runs with the advantage that the previous optimizations have already paved the path toward the optimum, as the electrostatic environment can be composed from the basic dipole considered here to get a first guess for the optimization parameters. A quantitative comparison with the exciting experiments in the field of $\mathrm{H}_{2}$ embedding in cavities [7,9] or $\mathrm{H}_{2}$ bridges [11] would need more realistic material specifications and corresponding modeling. In any event, the striking resemblance of our binding-energy curves in Fig. 4 with the theoretical curves related to the cavity experiments [7] deserves closer consideration. Our results already show that the simple configuration of two point charges can mimic the trapping of the $\mathrm{H}_{2}$ molecule found in a cavity. The critical distance and the amount of binding energy differ by a factor of 2, which can be imagined to be due to the details of the electrostatic field distributions and the caveat of the distance definition of realistic corrugated tips to be modeled by point charges.

In terms of solid or liquid media, one could associate the negative or positive tip charge situation with an anionic or cationic environment of a neutral $\mathrm{H}_{2}$ molecule that acts differently on the probability distribution of its nuclear configuration, i.e., forcing its direction parallel or perpendicular to the line connecting two opposing neighboring charges. It would need, besides the equal charge configuration considered here, also charges of different signs, costing a similar amount of computational time but with the additional complication that one would have to find the interesting and at least locally stable configurations among a large variety of configurations.

In summary, in a rather simple stable behavior, a hydrogen molecule between two equally charged tips adjusts itself so as to let the protons follow the external force, be it repulsion by positive charges or attraction by negative charges, thereby incorporating a rotation of the axis direction into a homogeneous distribution close to the equatorial plane in the former case and along the poles in the latter case. This picture should apply to more complicated charge embedding of the molecule as well. The vibrational quantum eigenstates are complicated by the rotational polar angle degree of freedom coherently mixed into radially quantized states after losing spherical symmetry due to the tips. However, clear fingerprints of stretching modes are observed as breathing modes in a spheroidal distribution of the axis direction. We are not aware that such properties could have been resolved in the BO approximation.

\section{ACKNOWLEDGMENTS}

This work has been supported in part by the Basque Departamento de Educación, Universidades e Investigación, the University of the Basque Country UPV/EHU (Grant No. IT1246-19), and the Spanish Ministerio de Ciencia y 
Tecnología (Grants No. FIS2016-76471-P and No. FIS201783780-P). The assistance of the DIPC computer center is gratefully acknowledged. W.S. is indebted to the Institute of Theoretical Physics and Astrophysics of CAU Kiel as well as to the university's computer center. M.A.V.H. acknowledges financial support from the Collaborative Research Fund of the Research Grants Council of Hong Kong (Grant No. C201415G). I.C.T.S. is supported by the HKBU Institute of Creativity, which is sponsored by the Hung Hin Shiu Charitable Foundation.
[1] J.-M. Lehn, Supramolecular Chemistry: Concepts and Perspectives (Wiley-VCH, Weinheim, 1995).

[2] R. A. Bissell, E. Córdova, A. E. Kaifer, and J. F. Stoddart, Nature (London) 369, 133 (1994).

[3] C. Cheng, P. R. McGonigal, S. T. Schneebeli, H. Li, N. A. Vermeulen, C. Ke, and J. F. Stoddart, Nat. Nanotechnol. 10, 547 (2015).

[4] L. Grill, K.-H. Rieder, F. Moresco, G. Rapenne, S. Stojkovic, X. Bouju, and C. Joachim, Nat. Nanotechnol. 2, 95 (2007).

[5] T. Kudernac, N. Ruangsupapichat, M. Parschau, B. Macia, N. Katsonis, S. R. Harutyunyan, K.-H. Ernst, and B. L. Feringa, Nature (London) 479, 208 (2011).

[6] G. Rapenne and C. Joachim, Nat. Rev. Mater. 2, 17040 (2017);. Nat. Nanotech. 12, 597 (2017).

[7] H. Wang, S. Li, H. He, A. Yu, F. Toledo, Z. Han, W. Ho, and R. Wu, J. Phys. Chem. Lett. 6, 3453 (2015).

[8] C. Lotze, M. Corso, K. J. Franke, F. von Oppen, and J. I. Pascual, Science 338, 779 (2012).

[9] E. Carbonell-Sanromà, M. Corso, J. Li, A. X. Brión-Ros, D. Sánchez-Portal, and J. I. Pascual, Surf. Sci. 678, 189 (2018).

[10] R. H. M. Smit, Y. Noat, C. Untiedt, N. D. Lang, M. C. van Hemert, and J. M van Ruitenbeek, Nature (London) 419, 906 (2002).

[11] D. Djukic, K. S. Thygesen, C. Untiedt, R. H. M. Smit, K. W. Jacobsen, and J. M. van Ruitenbeek, Phys. Rev. B 71, 161402(R) (2005).
[12] A. Halbritter, P. Makk, Sz. Csonka, and G. Mihaly, Phys. Rev. B 77, 075402 (2008).

[13] M. L. Trouwborst, E. H. Huisman, S. J. van der Molen, and B. J. van Wees, Phys. Rev. B 80, 081407(R) (2009).

[14] W. Kolos and L. Wolniewicz, J. Chem. Phys. 49, 404 (1968).

[15] K. Pachucki and J. Komasa, J. Chem. Phys. 130, 164113 (2009).

[16] G. D. Dickenson, M. L. Niu, E. J. Salumbides, J. Komasa, K. S. E. Eikema, K. Pachucki, and W. Ubachs, Phys. Rev. Lett. 110, 193601 (2013).

[17] D. R. Yarkony, J. Phys. Chem. 100, 18612 (1996).

[18] B. T. Sutcliffe and R. G. Woolley, Phys. Chem. Chem. Phys. 7, 3664 (2005).

[19] N. M. Tubman, I. Kylänpää, S. Hammes-Schiffer, and D. M. Ceperley, Phys. Rev. A 90, 042507 (2014).

[20] M. Casula, C. Attaccalite, and S. Sorella, J. Chem. Phys. 121, 7110 (2004).

[21] W. Schattke and R. Díez Muiño, Quantum Monte-Carlo Programming: For Atoms, Molecules, Clusters, and Solids, Monograph (Wiley-VCH, Weinheim, 2013).

[22] J. Bardeen, Phys. Rev. Lett. 6, 57 (1961).

[23] J. Tersoff and D. R. Hamann, Phys. Rev. B 31, 805 (1985).

[24] G. K. Birkner and W. Schattke, Z. Phys. 256, 185 (1972).

[25] See Supplemental Material at http://link.aps.org/supplemental/ 10.1103/PhysRevB.101.195436 for wave-function parameters and for detailed curves of total energy vs tip distance for ground and excited states. 